\title{
Gaining a new respect for the other guys: Scripted scenarios for inter professional education in paediatric emergencies
}

\author{
Ann Lazarsfeld-Jensen PhD and Donna Bridges PhD \\ Affiliations: \\ Charles Sturt University, New South Wales, Australia.
}

\begin{abstract}
\section{Introduction}

This project aimed to find ways of staging and evaluating simulations as a tool of interprofessional education (IPE). A series of scripted paediatric emergencies was used to create an intense learning environment to explore knowledge exchange and shared learning between GP registrars and paramedics. Although simulation is a familiar tool in medical education, its use in IPE is difficult to facilitate because of its many dimensions.
\end{abstract}

\section{Methods}

A complex set of observational methods was utilised comprising contemporaneous focus groups, questionnaires, video observation and participant feedback. These multiple methods captured richly descriptive data through triangulation, to overcome the problem of simulation becoming a staged performance.

\section{Results}

The research demonstrated there is great scope for simulation as a tool of IPE despite the time and labour intensity. Participants reported an interesting, exciting, quality learning experience and deep immersion in the scenarios.

\section{Conclusion}

To achieve its full potential, future IPE simulation would need to be paired with instructional clinical workshops for the practice and evaluation of skills, and close collaboration with the participants' own medical educators. However the possibilities of enhancing IPE learning by immersing different groups of health care professionals in unfamiliar, scripted, scenarios are probably only limited by budgets and the imagination of educators.

\section{Keywords}

simulation; interprofessional education, paediatric emergencies, medical education, SMBE, observation

\section{INTRODUCTION}

Simulation based medical education (SBME) is a highly effective tool that provides opportunities for students to practice clinical skills and decision making in a safe environment $(1,2)$. Simulation as a teaching tool can be used at multiple levels. The most fundamental use of simulation in medical education, for the safe repetition of psycho motor skills, is well documented, but the more complex uses still need careful evaluation $(3,4)$. SBME enables students to learn from their achievements as well as their errors. Simulation answers some of the problems of limited clinical placements, the risks of practising on patients, 
and the structural and financial pressures of meeting medical education needs in Australia today (5). Simulation is a solution to educational issues such as there being fewer patients available for student practice, changes in societal acceptance of students learning on real people, and the challenges of finding clinical education sites appropriate for student learning (5).

Interprofessional education (IPE) is an initiative involving members of two or more health professions who come together in a learning environment for the purpose of improving health care services and patient safety (6-8). Learning between members of the interprofessional team is interactive and fosters collaborative practice, communication, and teamwork. It supports the development of students to become professionals who are committed to collaborative patient centred practice $(5,8)$. IPE is a necessary educational initiative because '[e]vidence indicates that teamwork and collaboration skills are not intuitive or always learned on the job' (7). Health care workers may also define teamwork and collaboration differently. Without IPE health care professionals risk undervaluing and misunderstanding the roles and contributions of others. IPE can 'bridge the differences created by the separate socialization of health professions'(9).

Complex higher order learning may enhance tolerance and reduce communication breakdown (7). Miscommunication between health care practitioners is generally accepted as being a main cause of patient injury (5). In rare events such as paediatric emergencies a variety of stressors can cause loss of focus, conflict and blame, potentially leading to medical error and sentinel events $(5,9-11)$.

Error management involves identifying and acknowledging mistakes, analysis of errors; determination of corrections; and reflection (2). Reduction of mistakes is a central goal of both SBME \& IPE. Within a simulated environment there is safety to learn from mistakes or to think through and rehearse rare critical events without danger to patients. With the addition of facilitated debriefing SMBE can become a powerful learning experience that trains professionals to become accountable for acquiring new knowledge and skills and to confront errors.

Ziv et al (2) proposes that successful assessment and development in simulation training begins with an authentic environment, in which the students can immerse themselves as though it is real. Therefore the simulation must include three integral aspects of authenticity: an appropriate physical environment; a human environment that includes diverse staff, patient and family members; and clinical pathologies that are effectively simulated. A fourth factor that Ziv advocates is encounter with error, such as surgical injury, drug reactions, wrong diagnoses, non-optimal patient care (2).

The purpose of this study was to understand the uses of simulation as a tool in interprofessional education for medical and allied health training. The objective was to use scripted scenarios and high fidelity manikins to engage and immerse various health professionals in a simulated environment and to compare their experience as both participants and observers using multiple qualitative observational methodologies.

\section{METHODS}

\section{Study Design}

This research design was multi method, qualitative and involved observation of simulated scripted scenarios.

\section{Setting}

This project was conducted at the Interprofessional Education Simulation Centre at Charles Sturt University, Australia. Simulated general practitioner surgeries were set up so that paediatric emergencies occurred in a low-tech non-emergency environment.

\section{Participants}

For the research event a GP medical educator from BME invigilated the one-day research workshop, and the sample included an interprofessional team of general practitioner (GP) registrars, paramedic and nursing students (some double degree students) and paramedic and nursing academic staff. There were a total of 16 participants: four GP registrars, a supervising community GP, six academic staff members from CSU who were either from the nursing or paramedic disciplines; and five students in the nursing and paramedic double-degree undergraduate programs at CSU.

The GPs for this project $(n=4)$ were recruited from a variable population of interns on placement with GP practices in the Western region of New South Wales. Recruitment occurred through the GP training organisation (BME) which partnered in this project, which has a specific interest in finding interprofessional education opportunities. The other participants were drawn from a convenience sample of third year nursing, paramedic or double-degree students who self-selected following electronic media invitations, recruited from the university $(n=5)$. Available staff from the paramedic program in the School of Biomedical Science also volunteered $(n=6)$. 
Based on the limitations for participation in simulation laboratories, and with the use of qualitative methodologies, it is concluded the sample size was sufficient to generate meaningful data to answer the research question.

\section{Instrumentation}

A set of scripted scenarios of paediatric emergencies was developed for clinical authenticity and interprofessional skills. Programmable high fidelity manikins were used as the paediatric patients. Interactive keypads (clickers) were used in conjunction with Likert-type scale questionnaires that were shown on a screen, to obtain instant assessment of the participants' sense of immersion. Observational methods utilised closed circuit, and video replay.

\section{Procedures}

All GPs participated in three levels of the simulation event: practice, observation and reflection. Practice was participation in the scenario, reflection occurred in a nurse educator led debriefing, and observation involved participation in a simultaneous focus group in break-out rooms as the scenario was in progress. Video observation by academics who had not participated, occurred two weeks later.

\section{Practice}

The central research method we employed was the staging of scripted scenarios of simulated paediatric emergencies. Each of the four scenarios involved GP registrars, paramedic and nursing students, paramedic and nursing academic staff. Two high fidelity manikins were programmed as patients in the simulated doctors' surgeries. Each simulation drew on psychomotor skills and clinical decision-making, with the new element of an interprofessional challenge developed out of the known elements of interprofessional competencies, most of which centre on communication $(4,8,12)$. Social conflict was introduced at random as a stressor on the team. These scripted events were designed to capture the authentic demands on clinical skills and competencies during an emergency situation and to provide opportunities for interprofessional learning by testing the focus, leadership and teamwork of the participants.

\section{During the scenarios the four participating GP} registrars were required to practice assessment and history taking for paediatric emergencies that could occur in both a GP surgery and emergency department settings. Paramedic and nursing practitioners and paramedic and nursing students played scripted 'acting' roles where they played the parts of colleagues working alongside the doctors as well as family members. The scenarios required the GPs to perform a wide range of clinical treatments including:

- airway management

- intravenous access

- intraosseous needle placement

- fluid resuscitation

- medication administration, and

- management of cardiac arrest.

Throughout each scenario, the GP team leader needed to provide effective leadership, interprofessional communication, and team management skills in order to meet the clinical objectives. Sound clinical judgement, along with the ability to make difficult critical decisions, was paramount to the successful progression of each scenario. The ambiguous stressors, intended to elicit a reaction from the participants, included:

- a nurse performing a near miss drug error and subsequently being unable to function

- impatient paramedics

- disgruntled and obstructive colleagues

- a clinically trained 'actor' who played a family member who made inappropriate treatment suggestions

- a cross-cultural challenge, and

- the unexpected death of a child

\section{Observation}

The IPE Simulation Centre at Charles Sturt University has a system of video recording which allows the streaming of events to break out rooms. Practice in the simulations was limited to only one or two GPs and a few other clinicians at a time, so those that were not participating were able to view the scenario via live streaming in breakout rooms.

The facilitated observations of the unfolding scenario by video streaming were designed to keep participants who were not involved in actual simulations engaged in the process. They also were designed to enable focus groups to occur during the live streaming of the simulations. Observers comprised GPs and paramedic and nursing students. They were asked to discuss the differences in learning and teaching between observation and participation. They could comment at any time during the session. The four focus groups were conducted while observing the scenarios, audio recorded, and later transcribed and analysed thematically.

Two weeks later the videos were made available to a focus group of academics using an observational guide which correlated with the prompts used in the video streamed focus group session. 


\section{Reflection and Debriefing}

The first reflective process involved providing the participants with interactive keypads (clickers) to ascertain the level of authenticity of the simulated scenarios and to gauge the level of participant immersion. We gave the participants clickers just as they came out of the simulated event and before the debriefing session. A short set of questions was screened on an overhead projector and participants used their clickers to give instant responses to the questions.

The second reflection phase involved the participants in a formal and supervised debriefing session. The effectiveness of simulation for IPE is reinforced through the debriefing process, which occurs immediately after the scenario and usually takes as long as the scenario itself. The debriefing was designed to be an important level of evaluation of the simulation by the participants. They were conducted in an atmosphere of psychological safety to allow open discussion. All debriefings were structured to enable participants to reflect on their performance and provide feedback on both interprofessional and clinical medical skills ( 1 ).

The debriefings were not observed in real time, but they were filmed and these were later viewed by a focus group of six CSU academics involved in the project. The final reflection phase involved a focus group of the six CSU academics. These focus group participants observed the digital recordings of the simulation scenarios and the digital recordings of the debriefing sessions, and the purpose of this session was to ascertain the observational focus of clinical instructors who had not experienced the real-time event.

\section{RESULTS}

The research found that IPE simulation scenarios achieved a strong immersion experience and that within the simulation setting there was a constructive breech of professional boundaries that created a supportive team dynamic.

\section{Clickers}

The clickers (interactive keypad) measured the levels of stress, anxiety, vulnerability, cooperation, conflict and self-assessment of appropriate skills levels. We found that in three of the four scenarios the participants measured authentic immersion in the simulation. A positive measure for teamwork and supportiveness in the presence of stress indicates that inter professional education is not impeded by the content or delivery of a scenario. Samples of the way the clickers captured data and generated results are shown below.

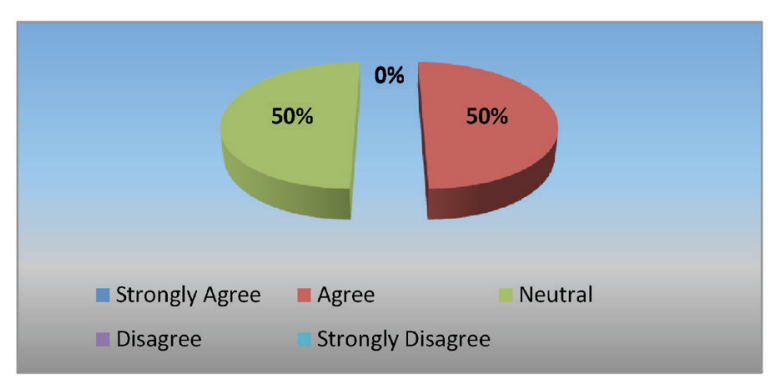

Figure 1: After each scenario a Likert-type scale was administered asking participants to respond to a set of statements. The statement measured here was / felt competent and in charge

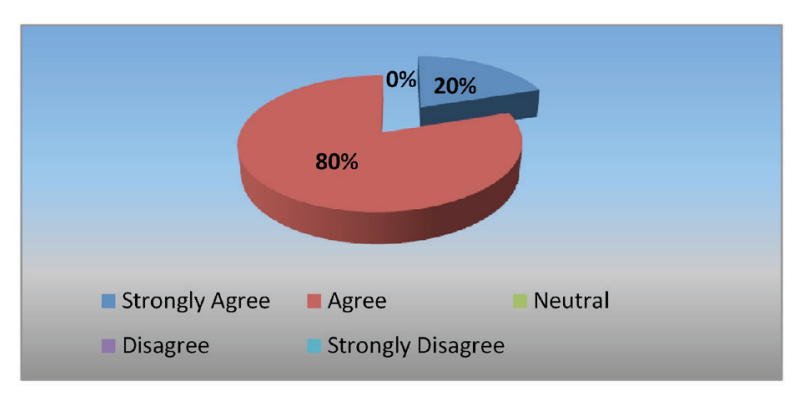

Figure 2 The statement measured here was / experienced some anxiety

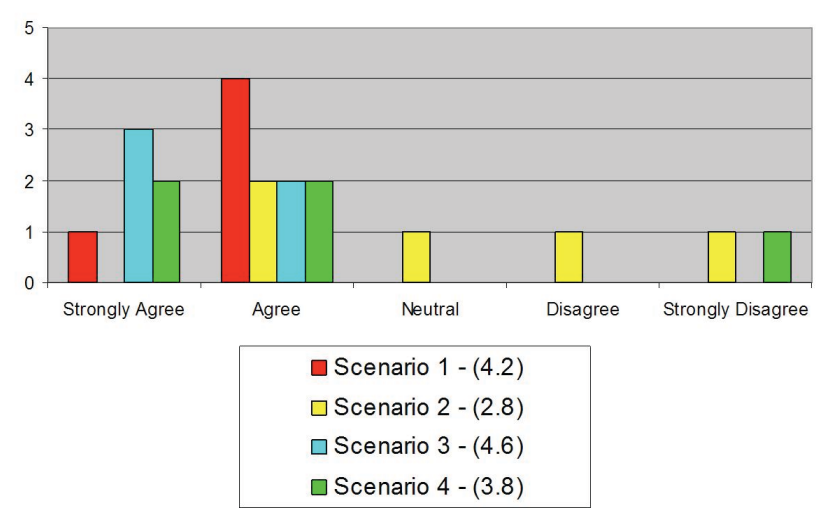

Figure 3: This compares responses to the statement $I$ experienced some anxiety for each scenario.

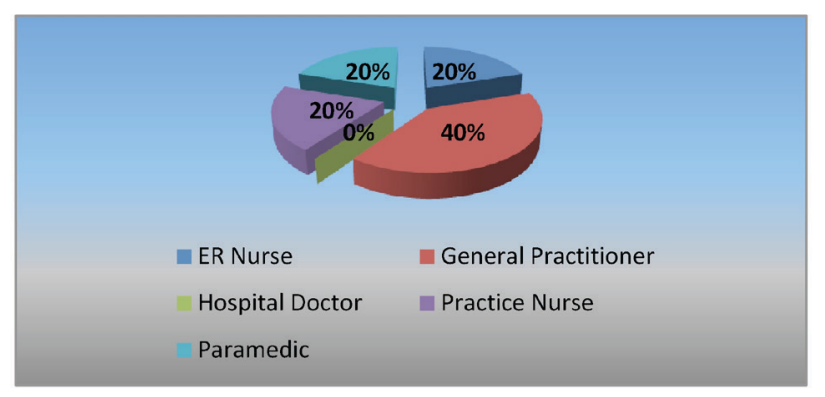

Figure 4: Clickers were also used for the collection of demographic data 


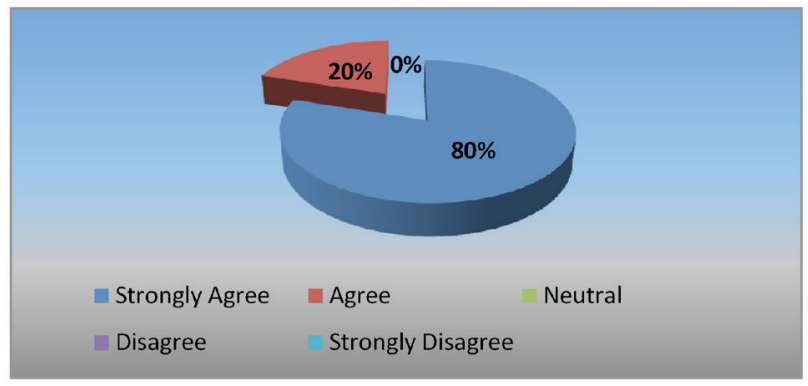

Figure 5: In this case there was an emphatic agreement with the statement I felt we formed a team.

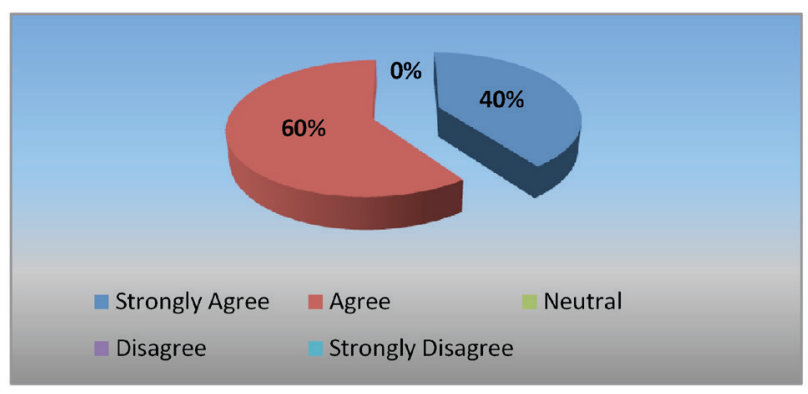

Figure 6: A less emphatic but still positive response from another team and scenario to I felt we formed a team

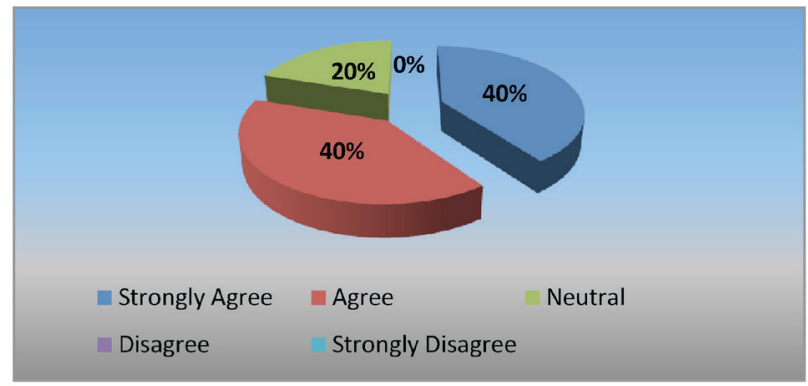

Figure 7: The statement / felt my skills were adequate, in one scenario produced $20 \%$ neutral responses, indicative of uncertainty.

\section{Debriefing}

The debriefing sessions held in-camera further validated the authenticity of the scenarios and demonstrated the level of interprofessional learning that occurred. In debriefings GP participants made statements that support the authenticity and immersion levels, such as:

'It nearly brought tears to my eyes' (GP3). 'I hope I never have to do that in my life' (GP1).

'It was a tough... It's not just the acute medicine - what do you say, what do you do, how do you phrase it - your child is dead' (GP2).

'It's a lot less stressful sitting here and watching it than being in it. But stress helps...it is a helpful thing at times' (GP3).
'The intensity of when I walked in there was huge. Physically when I went in there it was all quite overwhelming, I felt it going from this room to observing in the actual (clinical) space' (GP4).

\section{Observation}

We found that prior participation in a scenario was essential for effective IPE observation. Those who had not participated in a scenario but observed in either real time or video replay, tended to take a critical view of clinical skills rather than inter professional education. The difference in empathy was evidenced by these types of comments:

'He was depending on his knowledge of adult cardiac situations' (Student Observer 1).

'He was unsure of his knowledge and skills' (Academic Observer 1).

'But he did it so well and the mother just lost it, like started thumping on the chest and that. But he just stood there and then - yeah. But it was really good to - not really good, but it was good to see that' (GP Participant Observing).

'So yeah, it's absolute chaos...but you can sort of educate them on controlled chaos....you're capturing some of this unpredictability - this unpredictability is something that you learn from' (Student 2 Participant Observing).

\section{Focus Groups}

From the focus groups we found that the scenarios provided the opportunity to learn interprofessional skills of empathy, respect, teamwork, collaboration, problem solving and collaborative responses to a crisis.

'For me, when I heard the way you analyse things here,[during simulation] I have a great respect for you guys' (GP1 talking to paramedics).

'There are some people l've worked with out there who don't know how much you guys (paramedics) know or what you can do' (GP2 - talking to paramedics).

'We (paramedics) always sort of thought of doctors as God like. We didn't realise this (emergency medicine) isn't your bread and butter' (Student paramedic1).

Increased respect for the other professions was expressed by participants and the learning exchange confirmed there was mutual encouragement, transparency and an increased 
awareness of personal learning needs as well as better understanding of the skills and knowledge of the others. In addition the engaging nature of the event created opportunity for spontaneous and self-directed learning.

'I've found leadership is a challenge for me. And so I was actively watching how he was delegating' (GP3).

I was following $X$ (in the video) just because I was quite positively surprised by the way he communicated. He did give some feedback at times to the patient and he was speaking more clearly than everyone else, so I could hear him, and I guess that's why I followed him, naturally' (Student Paramedic 2).

\section{DISCUSSION}

This study successfully applied Ziv's (2) parameters to the development of scenarios that led to the level of immersion needed in simulation as a prelude to learning. Through the use of interactive keypads the researchers were confident that immersion had been achieved. Participants said they were genuinely stressed by the simulations, and this appropriately tested their psycho motor skills along with their problem solving and decision making capacity. As they were working in unfamiliar teams the elements of uncertainty and surprise pushed them into collaborative strategising, which is essentially inter professional. Moreover the sense of immersion, was transferred to their observation of others participating in other scenarios.

Uncertainty was most evident in the difficult scenario which involved a child death. The roleplaying mother was wearing a head covering and could not communicate a case history, although the child was known to a palliative care team. The mother was begging the doctors to save her child who was inert and asystole. Although this situation seemed extreme to some observers, it was in fact a reflection of a phenomena in some cultural groups where the family doctor is more trusted than uniformed paramedics who might take a family member away, or be unaware of cultural taboos. The scenario was harrowing for the participants who had not been warned that a child might die in the simulated surgery. All of the components of the scenario answered Ziv's (2) authenticity domains and were confirmed by debrief first-reaction comments such as: "Horror" and "I was lost" and comments on the leadership and collaborative aspects such as "I felt valued included," and "It was more collaborative than anything I have experienced in the field", and "You looked everyone in the eye and said - should we stop."
The IPE simulations created cognitive shifts for the practitioners involved that led to inter professional learning. Chaotic public spaces and brief interpersonal encounters are the milieu of paramedics, whereas GPs control their own spaces where they use a suite of inter-personal skills in disease prevention and management. Paramedics were surprised by the lack of high tech life support equipment in surgeries, and GP registrars were bewildered by some equipment that had been placed in the simulated surgeries. Nurses also practice in an environment that is more controlled with more resources and colleagues for support in decision-making. Both paramedics and GP registrars are more isolated and autonomous in their practice than nurses. The advanced life support skills that GPs find hard to acquire and maintain are also the core business of paramedic practice, yet on the other hand paramedics often have poor communication skills (13-15), while GPs excel in therapeutic conversation. Our presumption that knowledge exchange could be readily observed where two professions - GPs and paramedics - had such diverse strengths, was correct.

Comparing the experience of the real-time participants with that of the real-time observers, it became evident that observation was a potent learning tool for those who had also experienced a scenario. Individual participants were aware of their own strengths and weaknesses and used observation to learn from others, to evaluate their own performances, to rehearse and anticipate situations. Moreover those who had been in a scenario were empathetic and tolerant of the errors of others by comparison to the academic control group which tended to emphasise clinical competencies.

Participants expressed greater empathy than observers who had not previously participated. In the final scenario late in the day where immersion was less evident, participants felt there was a humorous sense of melodrama. The difference between types of observation were made clear when this video recording was observed by paramedic academics two weeks later, and they did not regard the scenario as any less authentic. The academics observing the recordings tended to have less empathy for perceived errors, and they focused critically on the psycho-motor skills of the GP registrars performing unfamiliar life support skills.

The focus of interest for individual observers was significant. Observers often made their own selection of which clinician to focus their observation on, or the clinical aspect that they wanted to watch. This was sometimes based on their personal learning needs, such as an interest in leadership or communication skills, or a 
decision about drugs dosage. As clinicians shared downtime from scenarios in the focusgroup their observations revealed the depth of learning which occurred spontaneously. In the focus groups the GPs were transparent about their own challenges to confidence and skills in emergency situations, and they expressed respect for the knowledge and skills of student paramedics. In addition, final year paramedic students discussed that their previous perception that doctors were 'god-like' and 'natural leaders' in emergency situations, when in fact the GPs said they were: "Waiting for you guys." The paramedics learned about the limitations of equipment in a general practice, the few opportunities doctors had to practice many of their skills, and the fact that general practitioners generally excelled in communications whereas those "hardwired for emergencies" would focus on hospital emergency department work.

To achieve its full potential future IPE simulation would need to be paired with instructional clinical workshops for the practice and evaluation of skills, and close collaboration with the participants' own medical educators. However the possibilities of enhancing IPE learning by immersing different groups of health care professionals in unfamiliar, scripted scenarios, are probably only limited by budgets and the imagination of educators.

This project was limited in that it was not designed to revisit the clinical skills deficits that participants identified during their debriefings. Moreover, debriefings were conducted by a nurse educator who did not feel comfortable discussing GPs skills, out of respect for the experimental nature of the events.

Simulation depends on technology and problems emerged such as an unfamiliar defibrillator that could not be made to work by participants, limited authenticity of the manikin in terms of pallor and breath sounds; the comparative weight and size of the manikin in relation to the child actor in a prequel video; the sudden substitution of manikins so that one was available for paramedics to transport.

\section{CONCLUSION}

This study aimed to understand the uses of simulation as a tool in interpersonal education for medical and allied health training. The research found that IPE simulation scenarios achieved a strong immersion experience. The research demonstrated there is great scope for simulation as a tool of IPE despite the time and labour intensity.

\section{CONFLICT OF INTEREST}

The authors declare they have no conflict of interests.

\section{ACKNOWLEDGEMENTS}

This research was made possible thanks to a grant from General Practice Education and Training (GPET) Australasia, in conjunction with Beyond Medical Education (Bathurst). A large team of academics and technical staff was involved in the production of these simulation scenarios, the videos and data collection. Special thanks to Craig Nelson, Dr Kam Wong, Joe Acker, John Rae, Tania Johnston, Brian Haskins, Amanda Hlushak, students, academic and technical staff the School of Biomedical Science, Charles Sturt University.

\section{REFERENCES}

1. Berkenstadt H, Erez D, Munz Y, Simon D, Ziv A. Training and assessment of trauma management: the role of simulation-based medical education. Anesthesiology Clinics. 2007;25(1):65.

2. Ziv A, Ben-David S, Ziv M. Simulation based medical education: an opportunity to learn from errors. Medical Teacher. 2005;27(3):1939.

3. McGaghie WC, Issenberg SB, Petrusa ER, Scalese RJ. A critical review of simulationbased medical education research: 20032009. Medical Education. 2010;44(1):50-63.

4. Bandali K, Parker K, Mummery M, Preece M. Skills integration in a simulated and interprofessional environment: An innovative undergraduate applied health curriculum. Journal of Interprofessional Care. 2008;22(2):179-89.

5. Robertson J, Bandali K. Bridging the gap: Enhancing interprofessional education using simulation. Journal of Interprofessional Care. 2008;22(5):499-508.

6. Ginns P, Kitay J, Prosser M. Developing conceptions of teaching and the scholarship of teaching through a Graduate Certificate in Higher Education. International journal for academic development. 2008;13(3):175-85.

7. Barnsteiner JH, Disch JM, Hall L, Mayer D, Moore SM. Promoting interprofessional education. Nursing Outlook. 2007;55(3):14450.

8. Bridges DR, Davidson RA, Odegard PS, Maki IV, Tomkowiak J. Interprofessional collaboration: three best practice models of interprofessional education. Medical education online. 2011;16. 
9. Developing Conceptions of Teaching and the Scholarship of Teaching through a Graduate Certificate in Higher Education.

10. Makary MA, Sexton JB, Freischlag JA, Holzmueller CG, Millman EA, Rowen L, et al. Operating room teamwork among physicians and nurses: teamwork in the eye of the beholder. Journal of the American College of Surgeons. 2006;202(5):746-52.

11. Valentin A, Capuzzo M, Guidet B, Moreno RP, Dolanski L, Bauer $P$, et al. Patient safety in intensive care: results from the multinational Sentinel Events Evaluation (SEE) study. Intensive care medicine. 2006;32(10):1591-8.

12. Buring SM, Bhushan A, Broeseker A, Conway S, Duncan-Hewitt W, Hansen L, et al. Interprofessional education: definitions, student competencies, and guidelines for implementation. American journal of pharmaceutical education. 2009;73(4).

13. Lazarsfeld-Jensen A. Starting young: the challenge of developing graduates' road readiness. Journal of Paramedic Practice. 2010;2(8):270-4.

14. Willis E, O'Meara P, Lazarsfeld Jensen A, McCarthy C. Paramedic education: developing depth through networks and evidence-based research. ALTC Online: www.altc.edu.au2009.

15. Willis E, Williams B, Brightwell R, O'Meara P, Pointon T. Road-ready paramedics and the supporting sciences curriculum. Focus on Health Professional Education: A Multidisciplinary Journal. 2010;11(2):1.

16. Fanning RM, Gaba DM. The role of debriefing in simulation-based learning. Simulation in Healthcare. 2007;2(2):115.

17. Knowles M. The adult learner: A neglected species: Gulf Publishing; 1978. 